\title{
Controlling Mercury Spills in Laboratories with A Thermometer Exchange Program
}

Lawrence D. McLouth, CIH

Environment Health and Safety Division

Lawrence Berkeley National Laboratory

1 Cyclotron Road

Berkeley, CA 94720

\section{$\underline{\text { Introduction }}$}

Mercury spills are common occurrences in laboratories. Though apparatuses such as barometers and manometers may be involved, most often spills are the result of broken mercury thermometers. Thermometers are normally broken by either dropping them on hard surfaces or by shearing them from baths, heating blocks and incubators. Because thermometers contain gram quantities of mercury they have the potential to contaminate large areas. This can result in personnel exposure to mercury and environmental pollution and often requires time-consuming clean ups.

Mercury toxicity and its effects on humans and the environment are common knowledge. Personnel exposure can occur through several routes including inhalation, dermal contact and ingestion. Though mercury exposure to laboratory personnel is a concern, of equal importance is the potential exposure to clean up personnel who have more intimate contact with contamination and debris.

Mercury spill cleanup requires expertise and equipment that is normally beyond the capabilities of most laboratories. Though laboratory personnel often clean minor spills of laboratory chemicals such as acids, bases and solvents, thorough mercury spill 
clean up requires special materials and monitoring equipment that are not part of most laboratories' arsenal of spill cleanup equipment. Consequently, intervention by industrial hygienists or other specially trained personnel is normally required.

Spills and their resulting clean ups may also interrupt laboratory work activities. At a minimum, the affected work area and sometimes even an entire laboratory needs to be secured to control personnel exposure and to minimize the spread of mercury to other non-contaminated areas. This invariably requires the cessation of laboratory activities in the affected area until the mercury can be cleaned and decontaminated. Moreover, spill clean up and waste disposal can be time-consuming and costly.

Though practices and procedures aimed at the careful handling of mercury thermometers can be developed, the most effective means of control is by either minimizing their use or eliminating them altogether.

The intent of this article is to present a case for replacing mercury thermometers with their organic-liquid-filled counterparts. A review of liquid-in glass-thermometers is given. In addition, a brief summary of mercury's health effects and exposure limits is presented. Spill cleanup methods and some lessons learned from our experience are offered as well. Finally, an overview of the mercury thermometer exchange program developed at Lawrence Berkeley National Laboratory (Berkeley Lab) is presented.

\section{Liquid-in-Glass-Thermometers}

A thermometer is an instrument that measures the intensity of heat in a known and reproducible manner. A "liquid-in-glass" thermometer consists of a closed glass tube (stem) filled with a liquid and a gas. Three common types of liquid-in-glass- 
thermometers include certified standard thermometers, working thermometers and general-purpose thermometers. Certified standard thermometers normally serve as primary and secondary standards for calibrating other thermometers ${ }^{1}$. These are calibrated using an approved method such as the American Society for Testing and Materials (ASTM) E- $77^{2}$. Certified standard thermometers are traceable to recognized standards organizations such as the National Institute of Standards and Technology (NIST) and should have a Report of Calibration ${ }^{1}$.

Working thermometers are used for tests and other applications where accurate temperature measurements are critical. These are verified against certified standard thermometers and normally have unique serial numbers so that they may be traced back to the standard.

General-purpose thermometers are used for common laboratory applications where accurate temperature measurements are not as critical. These are manufactured to Scientific Apparatus Manufacturer's Association/American National Standards Institute specifications ${ }^{1,3}$. These can be verified against certified standard thermometers for a given temperature or range as well.

The type of liquid used in thermometers depends on a number of factors including the temperature range of the thermometric system, the desired tolerance and repeatability, response time, cost and safety and environmental considerations. Two commonly used materials include mercury and organic liquids. The advantages and limitations of each type are discussed below. Table 1 summarizes their performance and working temperature ranges. 
Mercury has a linear coefficient of thermal expansion (i.e., its rate of expansion is relatively uniform over a broad temperature range). Accordingly, mercury-filledthermometers are reliable and provide good tolerance and repeatability ${ }^{4}$. Tolerance is defined as the extreme values permitted by regulation or specification of the difference between the indication of a thermometer and the true value ${ }^{5}$ (i.e., tolerance is the maximum permissible error of a thermometer). Repeatability is the closeness of agreement among the results of successive measurements of the same temperature carried out under the same conditions of measurement ${ }^{5}$. Mercury thermometers have relatively quick response times, coming to temperature within about three minutes. Their usable range is from -40 to $500^{\circ} \mathrm{C}$. The use of thallium and gallium amalgams can extend this range to -58 and $650^{\circ} \mathrm{C}$, respectively ${ }^{4}$. However, a major drawback of mercury-filledthermometers is that they can release toxic mercury to the environment if broken. This makes the use of organic-liquid-filled-thermometers an attractive alternative.

A number of different materials may be used for organic-liquid-filledthermometers. These include mineral spirits, toluene and citrus-based solvents, all of which are treated with dyes to permit visibility ${ }^{4}$. These materials tend to wet the borehole of the thermometer stem and tend to take longer times, up to fifteen minutes, to come to temperature. Also, they exhibit non-linear thermal expansion. The usable range is from -90 to $260{ }^{\circ} \mathrm{C}$. The lower end of the range for organic-liquid-filled-thermometers can be lowered to $-200^{\circ} \mathrm{C}$ by using pentane ${ }^{4}$. 


\section{The Health Effects of Mercury}

The routes of exposure to inorganic mercury include inhalation, skin absorption and ingestion. The acute effects of mercury exposure include pneumonitis, pulmonary edema, gingivitis, stomitis and renal disorders. Chronic effects normally involve central nervous system disorders such as fine tremors, ataxia and exaggerated reflexes.

Several Occupational Exposure Limits (OELs) have been established for limiting exposure to airborne inorganic mercury vapors. The federal Occupational Safety and Health Administration Permissible Exposure Limits (OSHA PEL) is 0.1 milligrams per cubic meter of air $\left(\mathrm{mg} / \mathrm{m}^{3}\right)$. This is a "Ceiling" limit, which must not be exceeded during any part of the workday. The American Conference of Governmental Industrial Hygienists Threshold Limit Value (ACGIH TLV ${ }^{\mathrm{TM}}$ ) is $0.025 \mathrm{mg} / \mathrm{m}^{3}$. This is an eighthour-time-weighted-average. The National Institute for Occupational Safety and Health (NIOSH) established three Recommended Exposure Limits for inorganic mercury. These include a $0.05 \mathrm{mg} / \mathrm{m}^{3}$ eight-hour-time-weighted-average, a $0.1 \mathrm{mg} / \mathrm{m}^{3}$ Ceiling and a 10 $\mathrm{mg} / \mathrm{m}^{3}$ Immediately Dangerous to Life or Heath (IDLH) level. An IDLH is an atmospheric concentration of any toxic, corrosive or asphyxiant substance that poses an immediate threat to life or would cause irreversible or delayed adverse health effects or would interfere with an individual's ability to escape from a dangerous atmosphere. The ACGIH and NIOSH have both given inorganic mercury a "Skin Notation", indicating that skin absorption is a route of exposure. It is important to note that the federal OSHA PEL is a legally enforceable limit, whereas both the ACGIH and NIOSH criteria are recommended limits. These OELs are summarized in Table 2. 


\section{Spill Clean Up and Decontamination Methods}

The amount of mercury spilled and the physical layout of the affected area dictate the materials and procedures required for cleaning up mercury spills. There is enough mercury in a single thermometer to contaminate a large area. Mercury has a tendency to break up into tiny beads making it difficult to distinguish the mercury from household dirt and debris. This is exacerbated when the spill occurs in an area congested with equipment, cabinets and shelves. Therefore a considerable portion of the spill clean up is spent in tracking down the small mercury droplets.

Care must be taken to prevent contaminating other surfaces, equipment and personnel during spill clean up. For this reason, it is prudent to cordon off the area with barricade tape or by using signs to limit access and to prevent inadvertently spreading contamination to other areas. When first approaching the spill area, air monitoring should be carried out to ensure that airborne mercury vapor levels are below the OELs (see Table 2.) This should be done with a direct reading instrument that is specific for mercury vapor such as the Jerome ${ }^{\mathrm{TM}}$ Model 411 Mercury Vapor Analyzer (or its successor, the Model 431-X). These instruments employ a gold film sensor for the detection and accurate measurement of mercury vapor in the air and are more reliable than instruments that use ultraviolet detection. Ultraviolet detectors may respond to the presence of other airborne contaminants, such as organic vapors, which give erroneously high readings.

Personal Protective Equipment (PPE) should be selected on the basis of visual observation and airborne monitoring results. Normally a lab coat, nitrile gloves, booties and safety glasses are adequate. Tyvek ${ }^{\mathrm{TM}}$ coveralls may also be worn as an added 
measure of protection. These are recommended, especially for large spills. Using double gloves, and changing them frequently during the course of a clean up is highly recommended as the hands are most likely to get contaminated. Both the hands and feet should be monitored periodically for mercury contamination. Unintentional contamination of equipment, supplies and surrounding areas is likely unless these precautions are taken.

Respiratory protection is selected on the basis of air monitoring results. Our experience has shown that airborne mercury vapor levels measured directly above beads and pools of mercury (i.e., several inches) may be quite high, reaching $0.5 \mathrm{mg} / \mathrm{m}^{3}$. However, breathing zone concentrations are normally at or below $0.003 \mathrm{mg} / \mathrm{m}^{3}$ which corresponds to the sensitivity of the direct reading instrument. An inhalation hazard doesn't exist at these concentrations. Therefore, respirators normally aren't needed unless the spill is extensive or involves heated surfaces such as drying ovens and incubators.

Clean up materials normally include flashlights, adhesive tape, a hand pump, a vacuum cleaner specifically designed for mercury, cleaning and vapor suppressing solutions, paper towels and plastic bags to contain the waste. These should be preassembled in a spill kit. It is best to use an impervious case as they are easy to clean and decontaminate. Fabric satchels should be avoided because they are easily contaminated and difficult to clean. Flashlights are useful for visually detecting mercury beads, especially small droplets. Adhesive tape can be used for picking up small droplets. A small hand operated pump equipped with a reservoir to hold mercury is effective for picking up larger beads (see Figure 1). The pump should be sealed in a plastic bag when 
not in use to minimize the spread of mercury contamination. For more extensive clean ups, a vacuum cleaner equipped with a mercury separator may be required (see Figure 2). These should be fitted with a Mersorb ${ }^{\mathrm{TM}}$ treated charcoal bed for adsorbing mercury vapors. Vacuum cleaners must be used slowly and methodically. A good approach is to vacuum one square foot (e.g., one standard 12"x12" floor tile) at a time. (Note: Vacuum hoses may have areas were the beads collect, especially in the older corrugated type hoses. Smooth lined hoses are best but even these should be replaced periodically.) Once visible beads have been removed from the contaminated surface, products such as Mercon $\mathrm{X}^{\mathrm{TM}}$ as well as Mercon ${ }^{\mathrm{TM}}$ spray and wipes are recommended for decontaminating affected areas and for suppressing mercury vapor (see Figure 3). These products contain reagents that form a non-volatile sulfide with mercury.

It is important to establish a release limit or a quantitative value to ascertain if an area has been effectively cleaned and decontaminated. The Berkeley Lab Industrial Hygiene Group adopted an airborne release limit of $0.003 \mathrm{mg} / \mathrm{m}^{3}$ for mercury. This is based on the sensitivity of the Jerome ${ }^{\mathrm{TM}}$ Models 411 and 431-X Mercury Vapor Analyzers and roughly corresponds to about one-tenth of the ACGIH TLV-TWA. The procedure used at Berkeley Lab is to first thoroughly clean and decontaminate the area and then measure the airborne mercury concentration. This is done indirectly by swiping the decontaminated area with an ordinary (dry) paper towel and measuring the emitted mercury vapor until several successive readings of $\leq 0.003 \mathrm{mg} / \mathrm{m}^{3}$ are attained (see Figure 4). This has proven to be a more sensitive method to confirm cleanliness than directly measuring the airborne mercury level above the decontaminated surface. It is 
thought that residual surface contamination, which ordinarily would not be detected with a direct reading instrument, is mechanically removed and concentrated on paper towels. Upon completion of clean up and decontamination activities, all spill clean up materials and debris should be put into labeled plastic bags and disposed of as mercurycontaminated waste. Reusable equipment, including mercury monitoring instrumentation should be swiped with paper towels and analyzed as previously described. It may be necessary to clean and decontaminate the equipment. Special attention should be given to the mercury vapor analyzer, especially its handle, which is particularly susceptible to accidental cross contamination. Clean up equipment such as small hand pumps should be sealed in a plastic bag. As previously stated, vacuum hoses may collect beads, especially in the old style corrugated hoses. Tapping the vacuum hose as it is held vertically, with the vacuum operating, will dislodge most of the beads adhering to the inner wall. Even so, the hose nozzle should be taped and sealed in a plastic bag when not in use. The case used to hold and transport clean up supplies should also be monitored with a mercury vapor analyzer to confirm that no cross contamination has occurred.

\section{Setting Up A Mercury Thermometer Exchange Program}

Developing and implementing a mercury thermometer exchange program is a relatively straightforward process. First it is necessary to communicate the impact of the problem (i.e., hazards, contamination and loss of productivity during clean up) to scientific staff. At Berkeley Lab, this was done by a site wide email followed up by several presentations given at various division and department staff meetings. Division personnel were informed that the focus of our program was to replace general-purpose thermometers with temperature ranges from -20 to $150^{\circ} \mathrm{C}$ and that participation was 
voluntary. (Note: certified standard and working thermometers as well as mercury containing equipment such as barometers and manometers were also included, but on a case by case basis). Some researchers were skeptical, arguing that we may be sacrificing performance for safety. They were informed, that while mercury thermometers do have better tolerances and quicker response times than their organic filled counterparts (Table 1), the latter could be verified over a desired temperature or a given temperature range. The slower response times of organic-liquid-filled-thermometers was not an issue for most applications.

Berkeley Lab's Environment Health and Safety (EH\&S) Division Waste Management Group procured bulk quantities of general-purpose thermometers. Safety personnel from scientific divisions gathered mercury thermometers in their areas. In most cases, this was done as part of their regular laboratory safety walkthroughs. The mercury thermometers were then exchanged with organic-liquid-filled-thermometers. The collected thermometers were sent to a facility for mercury reclamation at a nominal cost. Since intact thermometers are not classified as hazardous waste, there was no paperwork required from our scientific divisions. The only restriction imposed was that thermometers from radiologically controlled areas were required to be surveyed and released by the EH\&S Division Radiological Control Group prior to collection and exchange.

\section{Conclusion}

Mercury spills resulting from broken thermometers are common occurrences in laboratories. Because thermometers contain gram quantities of mercury, they can potentially affect relatively large areas, resulting in personnel contamination, 
environmental pollution and time-consuming clean ups. Since spill cleanup requires expertise and equipment that is normally beyond the reach of most laboratories, industrial hygienists or other specially trained personnel must often intervene. The resulting decontamination activities may interrupt laboratory work activities.

Though practices and procedures aimed at the careful handling of mercury thermometers can be developed, the most effective means of control is to either minimize their use or eliminate them altogether. This can be achieved by instituting an exchange program in which mercury thermometers are replaced with organic-liquid-filledthermometers. 
Table 1

Comparison of Mercury Filled vs. Organic-liquid-filled-thermometers ${ }^{\text {a }}$

\begin{tabular}{|c|c|c|c|}
\hline Thermometer Type & Usable Range ${ }^{\circ} \mathrm{C}$ & $\begin{array}{c}\text { Response Time } \\
\text { (Minutes) }\end{array}$ & Tolerance $\pm^{\circ} \mathrm{C}$ \\
\hline Mercury Filled & -40 to 500 & 3 & 1 \\
\hline $\begin{array}{c}\text { Organic Liquid } \\
\text { Filled }\end{array}$ & -90 to 200 & 15 & 3 \\
\hline
\end{tabular}

a. This data was obtained from the Miller Weber Inc. internet web site (reference 4) and applies to partial immersion thermometers.

Table 2

Occupational Exposure Limits $\left(\mathrm{mg} / \mathrm{m}^{3}\right)$ for Airborne Inorganic Mercury

\begin{tabular}{|l|c|c|c|}
\hline \multicolumn{1}{|c|}{ Source } & 8 Hour TWA & Ceiling & IDLH $^{\mathrm{d}}$ \\
\hline OSHA PEL $^{\mathrm{a}}$ & - & 0.1 & - \\
\hline ACGIH TLV $^{\mathrm{b}}$ & 0.025 (Skin) & - & - \\
\hline NIOSH REL $^{\mathrm{c}}$ & 0.05 (Skin) & 0.1 (Skin) & 10 \\
\hline
\end{tabular}

a. Federal Occupational Safety and Health Administration Permissible Exposure Limit

b. American Conference of Governmental Industrial Hygienists Threshold Limit Values

c. National Institute for Occupational Safety and Health Recommended Exposure Limits

d. Immediately Dangerous to Life or Health

e. A Skin notation indicates that skin absorption is a route of exposure. 


\section{Figure 1}

Hand-Operated Suction Pump for Small Droplets
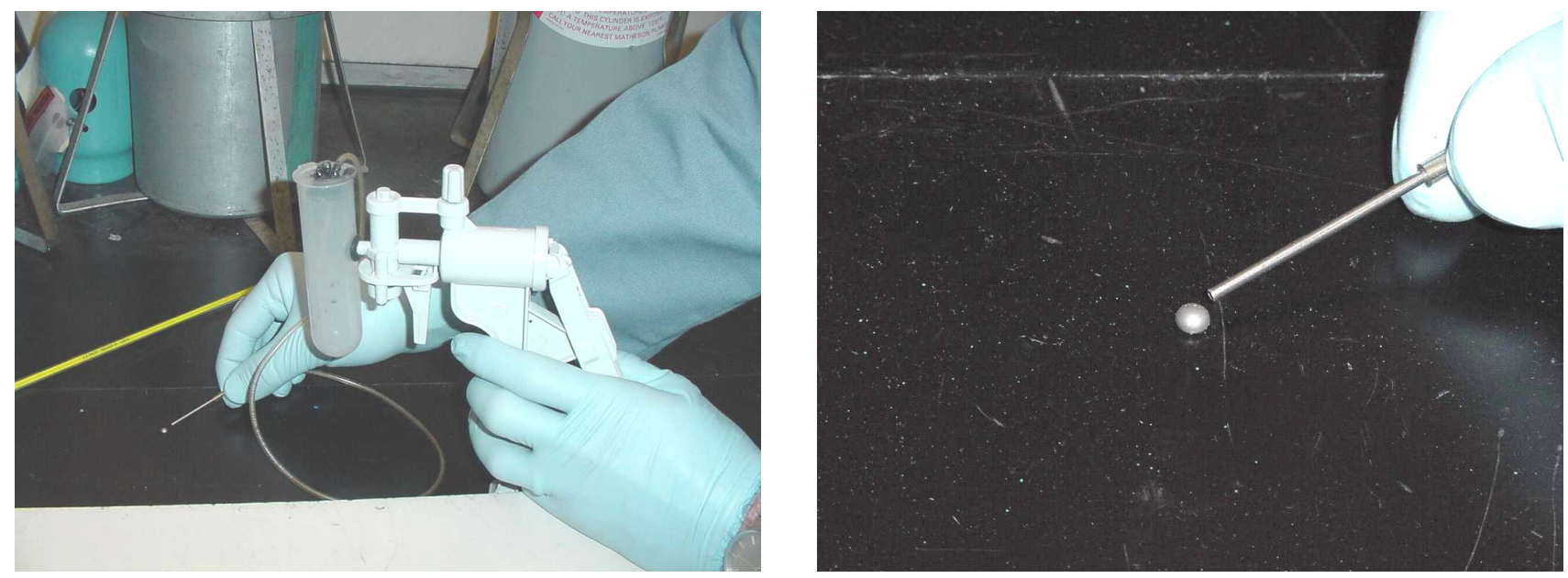

A small hand-operated pump equipped with a reservoir to hold mercury wastes.

Figure 2

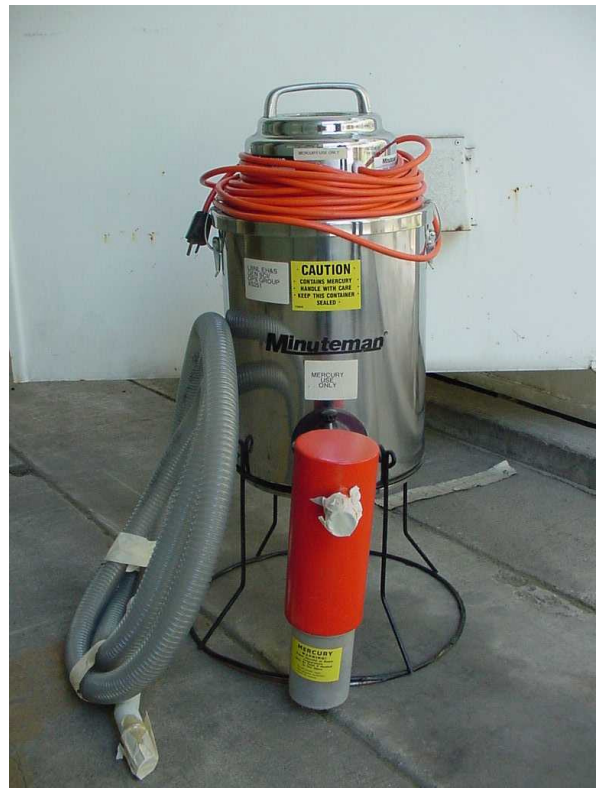

Mercury vacuum cleaner equipped with a mercury separator and a Mersorb ${ }^{\mathrm{TM}}$ treated charcoal bed. 
Figure 3

Mercury Vapor Suppressant Wipes

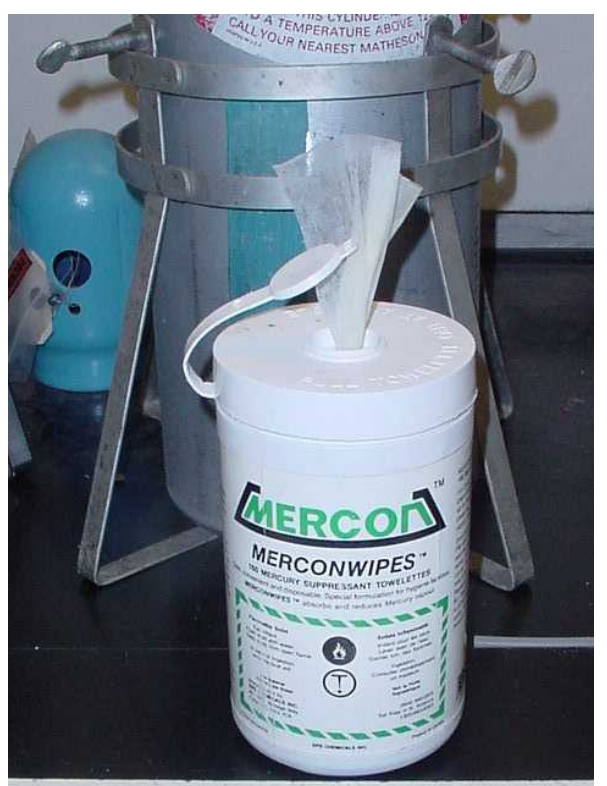




\section{Figure 4}

\section{Use of Towel to Remove Residual Mercury Contamination}

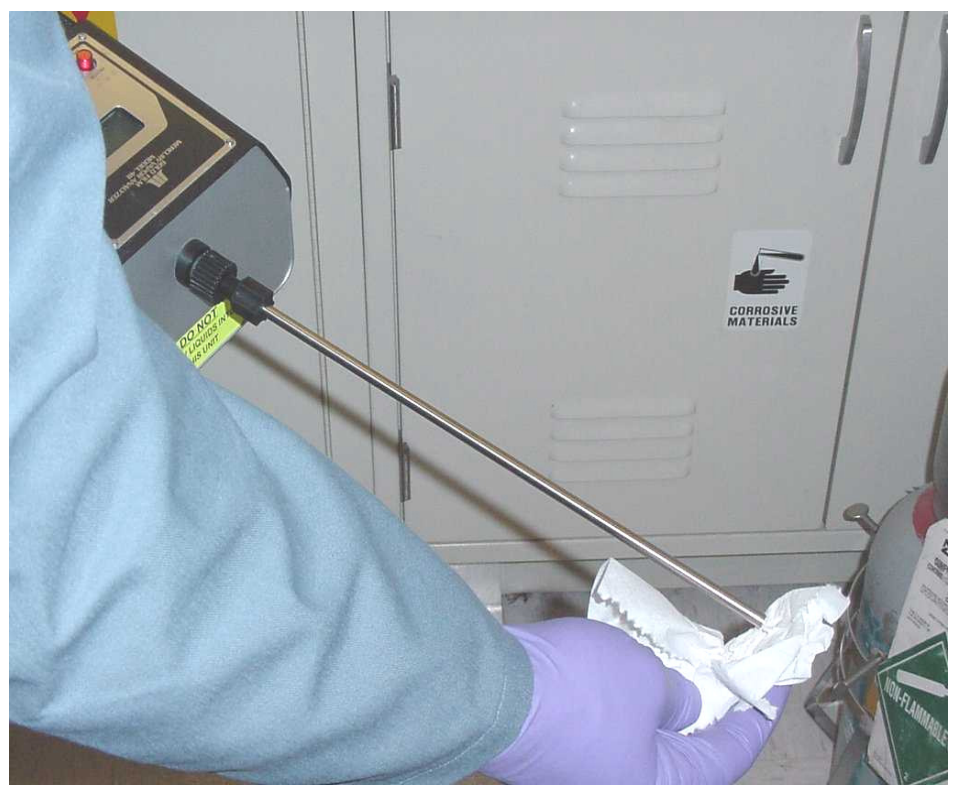

The airborne mercury vapor concentration is measured indirectly by swiping the

decontaminated area with a paper towel and using a direct reading instrument to measure the mercury vapor being "off gassed". 


\section{References}

1. Emory, D.M., American Laboratory News, Dec., 1998

2. ASTM E-77. 1995 Annual Book of ASTM Standards Section 14. Standard Test Methods for Inspection and Verification of Thermometers. CAmerican Society for Testing and Materials, 100 Barr Harbor Drive., West Conshohocken, PA 19428

3. SAMA/ANSI Z236.1-1986. American national standard for liquid-in-glass thermometers - general purpose laboratory use. US Dept. of Commerce, NIST Gaithersburg, MD.

4. Miller and Weber, Inc. internet web site: http://www.millerweber.com/index.htm 5. ASTM E-344. 1997 Annual Book of ASTM Standards Section 14.

Acknowledgements:

The author would like to thank Rob Connelly, CIH and Shelley Worsham, Environment Health and Safety Division, Lawrence Berkeley National Laboratory; Stephen Leland, IHIT, Office of Environmental Health and Safety, California State University, Sacramento; and Deanne M, Emory, Vice President, Miller and Weber, Inc. This paper would not have been possible without their input.

This work was supported by the Director, Office of Science, U.S. Department of Energy under Contract No. DE-AC03-76SF00098.

\section{DISCLAIMER}


This document was prepared as an account of work sponsored by the United States Government. While this document is believed to contain correct information, neither the United States Government nor any agency thereof, nor The Regents of the University of California, nor any of their employees, makes any warranty, express or implied, or assumes any legal responsibility for the accuracy, completeness, or usefulness of any information, apparatus, product, or process disclosed, or represents that its use would not infringe privately owned rights. Reference herein to any specific commercial product, process, or service by its trade name, trademark, manufacturer, or otherwise, does not necessarily constitute or imply its endorsement, recommendation, or favoring by the United States Government or any agency thereof, or The Regents of the University of California. The views and opinions of authors expressed herein do not necessarily state or reflect those of the United States Government

Ernest Orlando Lawrence Berkeley National Laboratory is an equal opportunity employer. 